\title{
Deriving the Suspended Backoff Counter Value Distribution in CSMA/CA
}

\author{
Raymond J Jayabal \\ School of Computer Engineering \\ Nanyang Technological University \\ Singapore 639798
}

\author{
Lau Chiew Tong \\ School of Computer Engineering \\ Nanyang Technological University \\ Singapore 639798
}

\begin{abstract}
Most of today's wireless LANs and PANs employ the use of the CSMA/CA protocol. In CSMA/CA, a randomly initialised counter is used to reduce the probability of nodes accessing the channel at the same time. In most implementations, this counter is suspended when the channel is idle. In this paper, we derive and verify through simulation the exact analytical expression for the distribution of this counter's value. For further validation, we show how it can be used to obtain the protocol's idle period distribution under saturation loads. For this latter distribution, we provide simulation results to show the accuracy of the formula compared to one derived using the de-facto first-order Markov channel state model.
\end{abstract}

\section{General Terms}

Medium Access Control (MAC), Analytical Modelling.

\section{Keywords}

Wireless data networks, LAN, PAN, CSMA/CA, random backoff algorithm.

\section{INTRODUCTION}

CSMA/CA is a MAC protocol that has widespread use. In the present day, one can find it ubiquitously operating in the IEEE 802.11 [1], Zigbee and HomePlug networking devices that have intruded deeply into the home, office and factory environments.

An important aspect of CSMA/CA is the use of a backoff counter to mitigate collision. As soon as it is ready to service the next frame from the upper protocol layers, the channel state is checked. If it is idle, the frame is transmitted at the start of the next available idle slot. If the channel is busy (i.e., with other nodes' transmissions), the backoff counter is initialised to a discrete value randomly uniform within the range $\{0, . . C W-1\}$, where $C W$ is a parameter known as the contention window length. When the channel becomes idle subsequently, the backoff counter is decremented at the end of each idle slot. When the backoff counter reaches zero, the frame is transmitted at the start of the next idle slot. If the transmission collides with other nodes' transmissions, the procedure is repeated again, with the backoff counter renewed to a random value in the said range twice wider depending on whether a fixed or exponentially increasing $C W$ is employed. If the channel becomes busy at any time before the backoff counter reaches zero, it is suspended.

To date, the distribution of the counter's value when this occurs is not known. Yet, knowledge of this fundamental parameter makes further analytical modelling of the protocol possible, especially in scenarios involving interferences from co-existing or exploitative systems which also attempt to use the shared channel for their own transmissions.
Take for example, the case where an aberrant node has joined a group of compliant nodes to transmit data over the shared channel. Assume that all nodes have a backlog of data to transmit (i.e. in saturation load), and that, in addition to abiding with the CSMA/CA protocol, the aberrant node also attempt to steal an extra transmission when the channel is sensed idle for longer than $k$ slots. Let $P\{W=w\}$ be the new backoff counter value probability mass function (PMF), $P_{C}(c) \equiv P\{C=c\}$ the single cell channel state PMF (more on this later), and $N$ the number of nodes including the aberrant node. With the suspended backoff counter PMF at our disposal, we can easily hypothesise that the probability that it will succeed in transmitting an extra frame during an idle period as:

$$
P_{s t}=\frac{\sum_{c=1}^{N}\left\{P_{C}(c) \cdot P\{W>k\}^{c} \cdot P\{F>k\}^{N-c}\right\}}{\sum_{c=1}^{N} P_{C}(c)}
$$

where the summed term in the nominator represents the probability that all nodes have their backoff counters at a value greater than $k$ following the occurrence of $c$ concurrent CSMA/CA-compliant transmissions, and $P\{F=f\}$ is the PMF of the suspended backoff counter value that we will derive and validate in this paper.

\section{RELATED WORK}

Over the years, many authors have contributed to further our understanding of the behaviour of the CSMA/CA protocol from an analytical perspective. Their studies have given us models for the protocol's transmission attempt probability, channel state and state transition probabilities and throughput under saturation loads.

Perhaps the most important contribution is from Bianchi $[2,3]$ with his introduction of the Markov chain modeling method. Prior to this method, analytical studies on the protocol were based on the p-persistent model, as contributed by H.S. Chhaya and S. Gupta in [4], and Cali et. al. in [5,6,7].

In Bianchi's model, the back-off counter is decremented right after a busy period. However, in actual implementation, it is only decremented at the end of an idle slot following that period. In [8], Ziouva and Antonakopoulos tried to correct this assumption, but did not take into account that only nodes that had transmitted in the previous busy period could start transmitting immediately after that period. In [9] and [10] done by Foh et al. and $\mathrm{Hu}$ et al., respectively, this shortcoming was addressed independently. 
Building on the results of these early works (mainly Bianchi's), two more characteristics - transmission delay and delay jitter were derived in [11] by Foh and Zuckerman, [12] by Chatzimisios et al., [13] by T. Sakurai and Hai L. Vu. and in [14] by Li et al.

To this body of work, we add our analysis on a perspective of the CSMA/CA protocol that have not been reported elsewhere: the probability mass function (PMF) of the suspended backoff counter value. In permutation with other known PMFs such as the initial backoff value and channel state PMFs, this distribution yields further understanding of the behaviour of the protocol. However, we restrict our analysis to a specific case of the CSMA/CA protocol whereby the contention window is fixed, because the steps involved in expanding it to the general case (i.e., whereby the contention window is incremented exponentially after each collision), eludes us. While this may seem like a significant limitation, it should be noted that this mode of operation has been shown in [15-17], to provide better performance than the exponential backoff scheme, especially when the contention window length is set optimally.

The rest of our paper is organized as follows: In Section 3, we review the protocol's channel state PMF which is required in our derivation. In Section 4, we present the steps in deriving the suspended backoff counter value PMF. In Section 5, we describe a sample application of this PMF which we will use for further validation of our analysis. In Section 6, we present a comparison of our hypothesis versus statistical results obtained from simulations. And finally, in Section 7, we conclude our paper.

\section{CHANNEL STATE DISTRIBUTION}

Let $C \in\{0,1, . . N\}$ be the discrete random variable representing the channel state for a cell with $N$ nodes, with $C=0$ representing idle state and $C=n$, the channel being busy with $n$ simultaneous transmissions. For CSMA/CA with fixed contention window length, the first-order Markov model for the channel state transition probabilities is given in [10] as follows:

$$
P_{C T}\left(c, c^{\prime}\right)= \begin{cases}\left(\begin{array}{l}
N \\
c
\end{array}\right)\left(\frac{2}{C W}\right)^{c}\left(\frac{C W-2}{C W}\right)^{N-c} & 0 \leq c \leq N, c^{\prime}=0 \\
\left(\begin{array}{l}
c \\
c^{\prime}
\end{array}\right)\left(\frac{1}{C W}\right)^{c}\left(\frac{C W-1}{C W}\right)^{c^{\prime}-c} & c \leq c^{\prime}, 1 \leq c^{\prime} \leq N \\
0 & c>c^{\prime}, 1 \leq c^{\prime} \leq N\end{cases}
$$

Here, $P_{C T}\left(c, c^{\prime}\right) \equiv P\left\{C_{i}=c \mid C_{i-1}=c^{\prime}\right\}$ with $c$ and $c^{\prime}$ being the current and previous states respectively. Using a transition matrix $\underline{P}$ with $(i, j)$ th elements equal to $P_{C T}(j, i)$, the channel state probability mass function $P_{C}(c) \equiv P\{C=c\}$ can be obtained via the steady-state equation,

$$
\underline{\pi}=\underline{\pi} \underline{P}
$$

with $P_{C}(i)$ given by the $(i)$ th element of $\underline{\pi}$.

\section{SUSPENDED BACKOFF COUNTER VALUE DISTRIBUTION}

Due to the second and third clauses in Eq.(2), note that consecutive busy states for $\mathrm{C}$ always follow the pattern $\left\{c_{0}, c_{l}\right.$, .., $\left.c_{k}, c_{k+1}, ..\right\}$, where $c_{k}>0, c_{k+1} \leq c_{k}$ for all $k$.

Consider the $\left(N-c_{0}\right)$ nodes that do not transmit at the onset of this interval of consecutive busy states. Each of them will have its backoff counter suspended at the same value at $c_{0}$ and every subsequent $c_{i}$ in the interval. Let $q\left(c_{0}\right)$ denote the average number of times each of these nodes suspends its backoff counter within all possible busy sequences which starts with state $c_{0}$. With $c_{0}=1$, and recalling that $1+a+a^{2}+\ldots=$ $1 /(1-a)$, the corresponding busy state sequences matching the pattern $\{1,1, . .1\}$ yields,

$$
q(1)=\frac{1}{1-P_{C T}(1,1)}
$$

With $c_{0}=2$, two busy state sequence patterns are possible: $\{2,2$, $. .2\}$ and $\{2,2, . ., 2,1, . ., 1\}$. It can be worked out that:

$$
q(2)=\frac{1}{1-P_{C T}(2,2)}+\frac{1}{1-P_{C T}(2,2)} \cdot P_{C T}(1,2) \cdot q(1)
$$

where the two terms are due to the two patterns, respectively. Working with upward values of $c_{0}$, we deduce that $q\left(c_{0}\right)$ is solvable with the recursive formula,

$$
q\left(c_{o}\right)= \begin{cases}\frac{1}{1-P_{C T}(1,1)} & c_{o}=1 \\ \frac{1+\sum_{i=1}^{c_{o}-1}\left\{P_{C T}\left(i, c_{o}\right) q(i)\right\}}{1-P_{C T}\left(c_{o}, c_{o}\right)} & c_{o}>1\end{cases}
$$

Over all possible busy state sequences, the average number of suspensions by the non-transmitting nodes can be expressed as the sum of $q\left(c_{0}\right)$ over all possible values for $c_{0}$, weighted by the product of the probability that the interval starts with $c_{0}$ and the number of nodes not transmitting in that interval, as follows:

$$
Q=\sum_{c_{0}=1}^{N} q\left(c_{0}\right) \cdot P_{C T}\left(c_{0}, 0\right) \cdot\left(N-c_{0}\right)
$$

Now consider the $c_{0}$ nodes that transmitted at the start of the interval. In any subsequent state $c_{i}$, should some of these nodes fail to select zero as their next backoff counter value, then $\left(c_{0}-\right.$ $c_{i}$ ) of these nodes would have their counters suspended at $c_{i}$ and subsequent states.

Denote $r\left(c_{0}\right)$ as the number of suspensions these nodes make in the interval which starts with $c_{0}$. Obviously, with $c_{0}=1$, no suspension will occur since a drop in the channel state marks the end of the interval, so $r(1)=0$. 
With $c_{0}=2$, only the busy pattern $\{2, . .2,1, . .1\}$ would result in one node repeatedly suspending its backoff counter. The average number of suspensions, given all possible sequences matching that pattern, would therefore be:

$$
r(2)=\frac{1}{1-P_{C T}(2,2)} \cdot P_{C T}(1,2) \cdot \frac{1}{1-P_{C T}(1,1)}
$$

With $c_{0}=3$, three patterns of busy state sequences result in counters being suspended: $\{3, . .3,2, . .2\},\{3, . .3,1, . .1\}$ and $\{3$, $. .3,2, . .2,1, . .1\}$. The first pattern will have one node repeatedly suspending its counter, while the last two will have 2 nodes doing so. Hence:

$$
\begin{aligned}
r(3)= & \frac{1}{1-P_{C T}(3,3)} \cdot P_{C T}(2,3) \cdot \frac{1}{1-P_{C T}(2,2)}+ \\
& 2 \cdot \frac{1}{1-P_{C T}(3,3)} \cdot P_{C T}(1,3) \cdot \frac{1}{1-P_{C T}(1,1)}+ \\
& 2 \cdot \frac{1}{1-P_{C T}(3,3)} \cdot P_{C T}(2,3) \cdot r(2)
\end{aligned}
$$

where each term is due to each of the three patterns. Working with upward values of $c_{0}$, we found that the recursive expression,

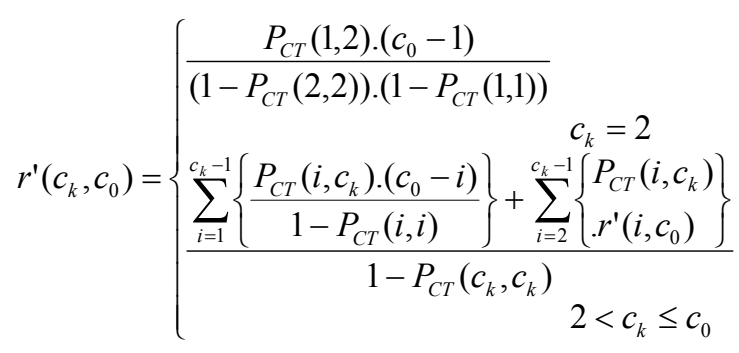

helps us solve $r\left(c_{0}\right)$ when we let $r\left(c_{0}\right)=r^{\prime}\left(c_{0}, c_{0}\right)$. Similar to Eq.(7), the average number of suspensions by transmitting nodes over all possible busy state patterns can be stated as:

$$
R=\sum_{c_{0}=2}^{N} r\left(c_{0}\right) \cdot P_{C T}\left(c_{0}, 0\right)
$$

Now let us consider the suspended counter value in relation to $Q$ and $R$ that we have derived above. Let the discrete random variables $F \in\{1,2, . . C W-1\}$ and $W^{\prime} \in\{1,2, . . C W-1\}$ represent the suspended counter value and the new non-zero value that the counter is initialised to. It should be noted that the event $\{F=f \mid$ $\left.W^{\prime}=f\right\}$ can only occur when a transmitting node selects a nonzero value as its new counter value during the consecutive busy state interval, since it will suspend its counter at that value. Over all possible values for $f$, therefore,

$$
\left.\sum_{f=1}^{C W-1} P_{F}(f)\right|_{W^{\prime}=f}=\frac{R}{Q+R}
$$

For nodes that do not transmit in the busy interval, their suspension values have to be at least one less than the newly assigned non-zero backoff value since the counter is decremented at every idle state and its occurence requires an idle to busy channel state transition. In other words, they can only contribute to the event $\left\{F=f \mid W^{\prime}>f\right\}$ occuring. Therefore,

$$
\sum_{w=2}^{C W-1} P\left\{F<w \mid W^{\prime}=w\right\}=\frac{Q}{Q+R}
$$

It is important to note that for each variate $w$ of $W^{\prime}, P\{F<w \mid$ $\left.W^{\prime}=w\right\}$ represents the sum of $(w-1)$ component probability terms $P_{F}(f) \mid W^{\prime}={ }_{w}$, one for each variate $f$ of $F$ satisfying the condition $f<w$. For example, $P\left\{F<w \mid W^{\prime}=3\right\}$ is the sum of $P_{F}(1) \mid W^{\prime}=3$ and $P_{F}(2) \mid W^{\prime}=3$. Furthermore, since the event of a non-transmitting node having its counter suspended is independent of the event of it selecting a particular variate of $W^{\prime}$, all the component probability terms of $P\left\{F<w \mid W^{\prime}=w\right\}$ are equal regardless of the value of $w$. As such, the following equation relating $P_{F}(f)$ for the 1st type of suspended backoff samples to the component probability $\left.P_{F}(f)\right|_{W^{\prime}=w, w>f}$ is valid:

$$
\begin{aligned}
& \left.P_{F}(f)\right|_{W^{\prime} \neq f} \\
& =\left.\sum_{w=f+1}^{C W-1} P_{F}(f)\right|_{W^{\prime}=w} \\
& =\left.P_{F}(f)\right|_{W^{\prime}=f+1}+\left.P_{F}(f)\right|_{W^{\prime}=f+2}+\ldots+\left.P_{F}(f)\right|_{W^{\prime}=C W-1} \\
& =\left.(C W-1-f) \cdot P_{F}(f)\right|_{W^{\prime}=w, w>f}
\end{aligned}
$$

Since the probability of suspensions in this case is equivalent to the sum of all the component probabilities across all possible values of $f$ and $w$ restricted to the condition $f<w$, we get the following expression relating it to the component probability:

$$
\begin{aligned}
& \sum_{w=2}^{C W-1} P\left\{F<w \mid W^{\prime}=w\right\} \\
= & \left.\sum_{f=1}^{C W-2} P_{F}(f)\right|_{W^{\prime} \neq f} \\
= & \left.\sum_{f=1}^{C W-2}(C W-1-f) \cdot P_{F}(f)\right|_{W^{\prime}=w, w>f} \\
= & \left.\{(C W-2)+(C W-3)+. .+1\} \cdot P_{F}(f)\right|_{W^{\prime}=w, w>f} \\
= & \left.\frac{(C W-1)(C W-2)}{2} \cdot P_{F}(f)\right|_{W^{\prime}=w, w>f}
\end{aligned}
$$

Eliminating the component probability term from Eqs.(14-15), we get:

$$
\left.P_{F}(f)\right|_{W^{\prime} \neq f}=\frac{2(C W-1-f)}{(C W-1)(C W-2)} \sum_{w=2}^{C W-1} P\left\{F<w \mid W^{\prime}=w\right\}
$$


Since $P_{F}(f)=\left.P_{F}(f)\right|_{W^{\prime}=f}+\left.P_{F}(f)\right|_{W^{\prime} \neq f}$, we can combine Eqs. $(12,13,16)$ to obtain the suspended backoff counter value PMF:

$$
P_{F}(f)= \begin{cases}\frac{R}{Q+R} & C W=2 \\
\frac{2(C W-1-f)}{(C W-1)(C W-2)} \cdot Q+\frac{1}{C W-1} \cdot R & C W>2 \\
\hline \begin{array}{l}
(17)
\end{array}\end{cases}
$$

\section{TEST APPLICATION: IDLE PERIOD DISTRIBUTION}

As a means to further validate the suspended backoff counter distribution, we apply to the calculation of the distribution of the idle periods for the protocol. This distribution can be used to determine the protocol's performance in the face of interference from a co-existing or exploitative system which also attempts to transmit when it senses the mutually shared channel as idle.

\subsection{Derivation Using The Suspended Backoff Counter Value Distribution}

With the probability mass functions $P_{F}(f)$ for the suspended backoff value at our disposal, we can express the probability mass function for the idle period given the preceeding channel state, $\left.P_{I}(i)\right|_{C=c}$ as the following permutation:

$$
\begin{aligned}
\left.P_{I}(i)\right|_{C=c}= & P\{W \geq i\}^{c} \cdot P\{F \geq i\}^{N-c} \times \\
& \left(1-\left(1-\left.P_{W}(i)\right|_{W \geq i}\right)^{c}\left(1-\left.P_{F}(i)\right|_{F \geq i}\right)^{N-c}\right)
\end{aligned}
$$

A bit of explanation may be necessary to induct the reader to how the above equation is derived: Note that for the idle period to be equal to $i$, both the new and suspended backoff counters must be equal to or higher than $i$, with at least one of them equalling i. So the first two factors involving the terms $P\{W \geq i\}$ and $P\{F \geq i\}$ ensures the former condition, while the remaining factor ensure the latter. For the complete idle period distribution, i.e., one that spans across the entire measured space, we perform a weighted summation of Eq.(18) over all channel states:

$$
P_{I}(i)=\frac{\sum_{c=1}^{N}\left\{\left.P_{C}(c) \cdot P_{I}(i)\right|_{C=c}\right\}}{\sum_{c=1}^{N} P_{C}(c)}
$$

\subsection{Derivation Using De-Facto Markov Model}

By way of Eq.(2), each variate $i$ of the distribution can be approximated as having the occurrence probability of a string of consecutive idle states of length $(I-1)$. Though this method is flawed since it ignores that the string length is bounded to $C W-$ 1 , to the best of our knowledge, it is the only one available currently:

$$
P_{I}(i)= \begin{cases}\frac{\sum_{c=1}^{N}\left\{P_{C}(c) P\left\{C>0 \mid C^{\prime}=c\right\}\right\}}{\sum_{c=1}^{N} P_{C}(c)} & i=0 \\
\frac{\left(\begin{array}{c}
\sum_{c=1}^{N}\left\{P_{C}(c) P\left\{C>0 \mid C^{\prime}=c\right\}\right\} \\
\times P_{C T}(0,0)^{i-1} P\left\{C>0 \mid C^{\prime}=0\right\}
\end{array}\right)}{\sum_{c=1}^{N} P_{C}(c)} & 0<i<C W\end{cases}
$$

\section{MODEL VERIFICATION}

The $n s-2$ [18] program was used to obtain the simulation results of the suspended backoff counter value $P_{F}(f)$ and idle period distribution $P_{I}(i)$ of the protocol. Nodes were placed arbitrarily within a $10 \mathrm{~m}$ by $10 \mathrm{~m}$ area with frame sizes set to a constant value (512 bytes), and fed with saturation loads. Changes were made to the IEEE 802.11 source code to ensure that the contention window is fixed, and to disable radio capture (since the focus of our analysis is purely on the CSMA/CA protocol).

For each combination of $C W$ and $N$, the program was run 25 times with each run using a different seed value for the random value generator and spanning 100,000 channel state transitions. In each run, the expectation and variance of the suspended backoff counter value and idle period was determined and recorded, such that in the end we had 25 samples of each value per combination of $C W$ and $N$.

To validate our model, we ran the one sample T- and Chi-square test. In the T-test, the $95 \%$ confidence interval $(95 \% \mathrm{CI})$ was calculated based on the 25 samples obtained per combination of $\mathrm{CW}$ and $N$, and compared against the analytical values given by Eqs.(16,20). In all combinations of $C W$ and $N$, the analytical values were well within the $95 \% \mathrm{CI}$ of the values obtained from the simulation.

We present several results here in brief. In Tables 1-4, the analytical results for the frozen back-off counter distribution expectation $(E[F])$ and variance $(\operatorname{Var}[F])$ with $N=\{2,4,7,10\}$ is shown to be within the $95 \% \mathrm{CI}$ of the simulation results across the range $2 \leq C W \leq 32$. In Figs. 1-4, the idle period distribution expectation $(E[I])$ and variance $(\operatorname{Var}[I])$ for $C W=\{4,8,16,32\}$ computed using Eq.(20) is seen to fall within the $95 \%$ CI of the simulation results across the range $2 \leq N \leq 10$. 
Table 1: Expectation \& Variance for the Frozen Back-off Distribution: Analytical versus Simulation (for $\mathbf{N}=2$ )

\begin{tabular}{|c|c|c|c|c|}
\hline $\boldsymbol{C W}$ & $\begin{array}{c}\text { E[F] } \\
\text { Ana. }\end{array}$ & $\begin{array}{c}\text { E[F] Sim } \\
(\mathbf{9 5 \%} \text { CI })\end{array}$ & $\begin{array}{c}\text { Var[F] } \\
\text { Ana. }\end{array}$ & $\begin{array}{c}\text { Var[F] Sim } \\
(\mathbf{9 5 \%} \text { CI })\end{array}$ \\
\hline 2 & 1.0000 & 1.0000 & 0.0000 & 0.0000 \\
& & 1.0000 & & \\
\hline 4 & 1.4444 & 1.4406 & 0.3580 & 0.3529 \\
& & 1.4535 & & 0.3638 \\
\hline 8 & 2.7143 & 2.7033 & 2.3469 & 2.3226 \\
& & 2.7272 & & 2.3707 \\
\hline 12 & 4.0303 & 4.0002 & 6.1203 & 6.0263 \\
& & 4.0383 & & 6.1803 \\
\hline 16 & 5.3556 & 5.3220 & 11.674 & 11.500 \\
& & 5.3725 & & 11.805 \\
\hline 20 & 6.6842 & 6.6491 & 19.006 & 18.743 \\
& & 6.7335 & & 19.340 \\
\hline 24 & 8.0145 & 7.9781 & 28.116 & 27.315 \\
& & 8.0686 & & 28.416 \\
\hline 28 & 9.3457 & 9.2937 & 39.004 & 38.647 \\
& & 9.4392 & & 39.604 \\
\hline 32 & 10.677 & 10.582 & 51.670 & 51.198 \\
& & 10.718 & & 53.058 \\
\hline
\end{tabular}

Table 3: Expectation \& Variance for the Frozen Back-off Distribution: Analytical versus Simulation (for $\mathbf{N}=7$ )

\begin{tabular}{|c|c|c|c|c|}
\hline $\boldsymbol{C W}$ & $\begin{array}{c}\text { E[F] } \\
\text { Ana. }\end{array}$ & $\begin{array}{c}\text { E[F] Sim } \\
(\mathbf{9 5 \%} \text { CI })\end{array}$ & $\begin{array}{c}\text { Var[F] } \\
\text { Ana. }\end{array}$ & $\begin{array}{c}\text { Var[F] Sim } \\
(\mathbf{9 5 \%} \text { CI })\end{array}$ \\
\hline 2 & 1.0000 & 1.0000 & 0.0000 & 0.0000 \\
& & 1.0000 & & \\
\hline 4 & 1.5097 & 1.5091 & 0.4263 & 0.4254 \\
& & 1.5134 & & 0.4301 \\
\hline 8 & 2.7398 & 2.7358 & 2.4119 & 2.4018 \\
& & 2.7504 & & 2.4306 \\
\hline 12 & 4.0423 & 4.0403 & 6.1673 & 6.1507 \\
& & 4.0585 & & 6.2137 \\
\hline 16 & 5.3623 & 5.3568 & 11.709 & 11.666 \\
& & 5.3805 & & 11.760 \\
\hline 20 & 6.6885 & 6.6678 & 19.034 & 18.864 \\
& & 6.7082 & & 19.108 \\
\hline 24 & 8.0176 & 7.9886 & 28.140 & 27.928 \\
& & 8.0335 & & 28.244 \\
\hline 28 & 9.3479 & 9.3154 & 39.024 & 38.839 \\
& & 9.3723 & & 39.293 \\
\hline 32 & 10.679 & 10.651 & 51.688 & 51.354 \\
& & 10.709 & & 51.893 \\
\hline
\end{tabular}

These tables and figures are but a sampling of the results of our tests. Over the range of values for $C W=\{2,3, . ., 32\}$ and $N=\{2$, .., 10$\}$, our hypothesis passed both the T- and Chi-square tests, suggesting that our analysis is indeed accurate. In addition, it can be seen in Figs. 1-4 that for small values of $N$, the approximation of $\operatorname{Var}[I]$ based on the Markov model given by Eq.(20) performs poorly compared to our model. This suggests that this de-facto model is not appropriate when used in scenarios involving small number of nodes, i.e., ranging from 2 to 10 .
Table 2: Expectation \& Variance for the Frozen Back-off Distribution: Analytical versus Simulation (for $\mathrm{N}=4$ )

\begin{tabular}{|c|c|c|c|c|}
\hline $\boldsymbol{C W}$ & $\begin{array}{c}\text { E[F] } \\
\text { Ana. }\end{array}$ & $\begin{array}{c}\text { E[F] Sim } \\
(\mathbf{9 5 \%} \text { CI) }\end{array}$ & $\begin{array}{c}\text { Var[F] } \\
\text { Ana. }\end{array}$ & $\begin{array}{c}\text { Var[F] Sim } \\
(95 \% \text { CI })\end{array}$ \\
\hline 2 & 1.0000 & 1.0000 & 0.0000 & 0.0000 \\
& & 1.0000 & & \\
\hline 4 & 1.4767 & 1.4749 & 0.3928 & 0.3898 \\
& & & & 0.3965 \\
\hline 8 & 2.7244 & 2.7207 & 2.3729 & 2.3724 \\
& & 2.7332 & & 2.3956 \\
\hline 12 & 4.0349 & 4.0149 & 6.1385 & 6.0901 \\
& & 4.0482 & & 6.1824 \\
\hline 16 & 5.3582 & 5.3364 & 11.687 & 11.566 \\
& & 5.3800 & & 11.761 \\
\hline 20 & 6.6859 & 6.6812 & 19.017 & 18.836 \\
& & 6.7296 & & 19.235 \\
\hline 24 & 8.0157 & 8.0047 & 28.125 & 27.837 \\
& & 8.0585 & & 28.303 \\
\hline 28 & 9.3465 & 9.2871 & 39.012 & 38.642 \\
& & 9.3605 & & 39.377 \\
\hline 32 & 10.678 & 10.631 & 51.677 & 50.945 \\
& & 10.728 & & 51.838 \\
\hline
\end{tabular}

Table 4: Expectation \& Variance for the Frozen Back-off Distribution: Analytical versus Simulation (for $N=10$ )

\begin{tabular}{|c|c|c|c|c|}
\hline CW & $\begin{array}{c}\text { E[F] } \\
\text { Ana. }\end{array}$ & $\begin{array}{c}\text { E[F] Sim } \\
(\mathbf{9 5 \%} \text { CI })\end{array}$ & $\begin{array}{c}\text { Var[F] } \\
\text { Ana. }\end{array}$ & $\begin{array}{c}\text { Var }[F] \text { Sim } \\
(\mathbf{9 5 \%} \text { CI })\end{array}$ \\
\hline 2 & 1.0000 & 1.0000 & 0.0000 & 0.0000 \\
& & 1.0000 & & \\
\hline 4 & 1.5292 & 1.5269 & 0.4450 & 0.4439 \\
& & 1.5313 & & 0.4478 \\
\hline 8 & 2.7545 & 2.7526 & 2.4487 & 2.4447 \\
& & 2.7597 & & 2.4587 \\
\hline 12 & 4.0499 & 4.0458 & 6.1970 & 6.1825 \\
& & 4.0590 & & 6.2234 \\
\hline 16 & 5.3667 & 5.3659 & 11.733 & 11.720 \\
& & 5.3854 & & 11.790 \\
\hline 20 & 6.6914 & 6.6806 & 19.053 & 18.970 \\
& & 6.7158 & & 19.137 \\
\hline 24 & 8.0194 & 7.9802 & 28.155 & 27.950 \\
& & 8.0299 & & 28.265 \\
\hline 28 & 9.3493 & 9.3197 & 39.038 & 38.801 \\
& & 9.3671 & & 39.271 \\
\hline 32 & 10.680 & 10.650 & 51.699 & 51.420 \\
& & 10.725 & & 51.946 \\
\hline
\end{tabular}

\section{CONCLUSION}

We have presented an analytical model for the suspended backoff counter distribution, backing it with simulation results which certify its correctness. The derivation of this distribution is not found elsewhere in the literature, and may be useful in further characterisation of the CSMA/CA protocol especially in scenarios involving interference from other systems or nonconformant nodes. As further demonstration to its accuracy, we also tested the correctness of this distribution when used to derive the protocol's idle period. In the process of doing so, found it not only to be correct, but also that the only method prior to the availability our model - i.e., the one deriving from the Markov model, is inaccurate and cannot be used for the number of nodes that is typical in real life scenarios. 


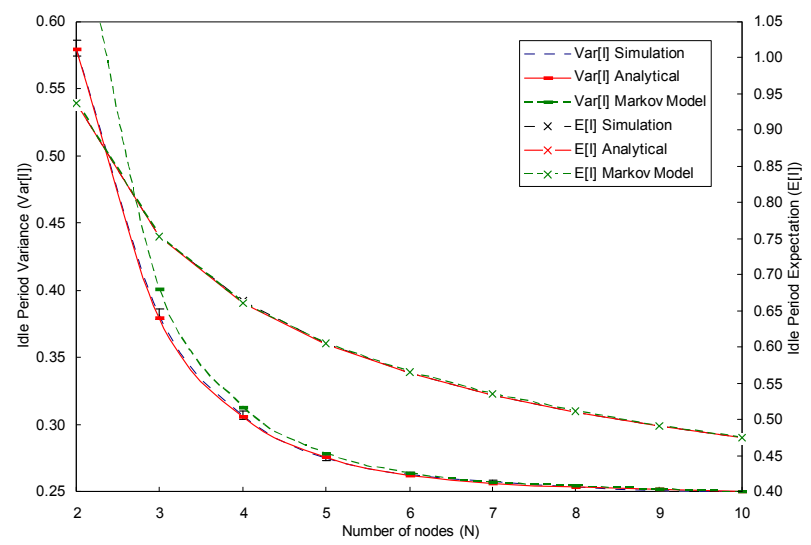

Fig. 1: Idle Period Expectation \& Variance: Analytical, Simulation and Markov Model (for $\mathrm{CW}=4$ ).

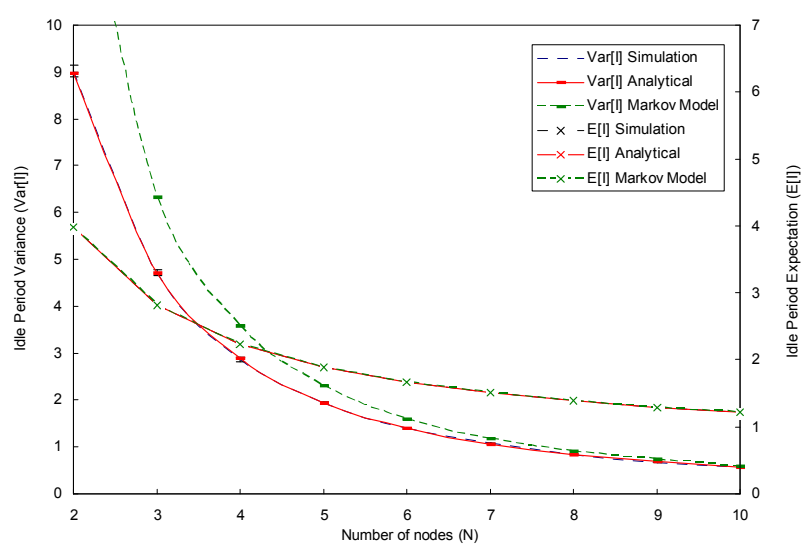

Fig. 3: Idle Period Expectation \& Variance: Analytical, Simulation and Markov Model (for $\mathrm{CW}=16$ ).

\section{REFERENCES}

[1] "IEEE Std. 802.11-2007, Part 11: Wireless LAN Medium Access Control (MAC) and Physical Layer (PHY) Specifications," IEEE Std. 802.11, 2007.

[2] G. Bianchi, "IEEE 802.11- Saturation Throughput Analysis", IEEE Comm. Letters, Vol. 2, No. 12, December 1998.

[3] G. Bianchi, "Performance Analysis of the IEEE 802.11 Distributed Coordination Function," in IEEE Journal on Selected Areas in Comm., Vol. 18, No. 3, March 2000, pp. 535-547.

[4] H. S. Chhaya, S. Gupta, "Performance Modeling of Asynchronous Data Transfer Methods of IEEE 802.11 MAC Protocol," in Wireless Networks 3 (1997), pp.217234, 1997.

[5] F. Cali, M. Conti, E. Gregori, "Dynamic Tuning of the IEEE 802.11 Protocol to Achieve a Theoretical Throughput Limit", in IEEE/ACM Trans. On Networking, Vol. 8, No. 6, December 2000.

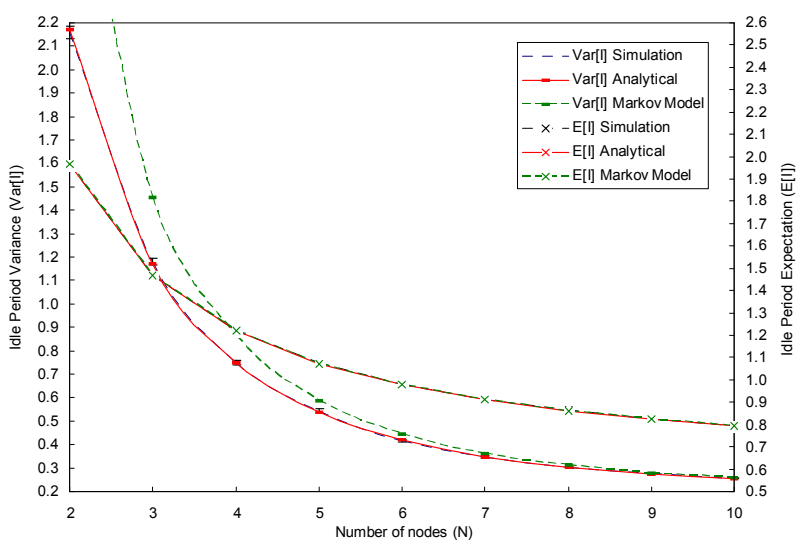

Fig. 2: Idle Period Expectation \& Variance: Analytical, Simulation and Markov Model (for $\mathrm{CW}=8$ ).

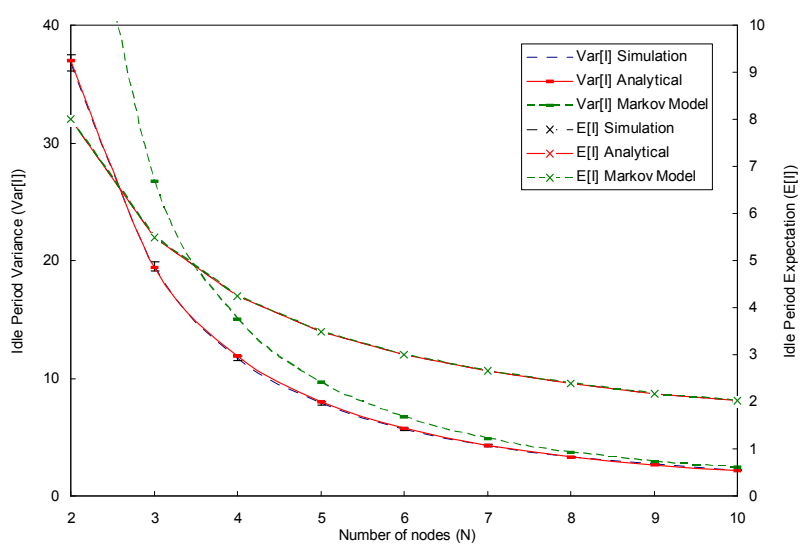

Fig. 4: Idle Period Expectation \& Variance: Analytical, Simulation and Markov Model (for $\mathrm{CW}=32$ ).

[6] F. Cali, M. Conti, E. Gregori, "IEEE 802.11 Protocol: Design and Performance Evaluation of an Adaptive Backoff Mechanism", in IEEE Journal on Selected Areas in Communications 2000, Vol. 18, No. 9, pp. 1774-1786.

[7] F. Cali, M. Conti, E. Gregori, "IEEE 802.11 Wireless LAN: Capacity Analysis and Protocol Enhancement", in Proceedings of IEEE INFOCOM'1998, March, 1998.

[8] E. Ziouva and T. Antonakopoulos, "CSMA/CA Performance under High Traffic Conditions: Throughput and Delay Analysis," in Computer Communications, Vol. 25, No. 3, February 2002, pp. 313-321.

[9] C. H. Foh and J. W. Tantra, "Comments on IEEE 802.11 Saturation Throughput Analysis with Freezing of Backoff Counters," in IEEE Communications Letters, Vol. 9, No. 2, 2005, pp. 130-132.

[10] C. Hu, H. Kim, and J. C. Hou. "An Analysis of the Binary Exponential Backoff Algorithm in Distributed MAC Protocols," in Tech. Rep. No. UIUCDCS-R-2005-2599 UILU-ENG-2005-1794. July 2005. 
[11] C. H. Foh and M. Zukerman, "Performance analysis of the IEEE 802.11 MAC protocol" in European Wireless 2002, February 2002, Florence, Italy.

[12] P. Chatzimisios, V. Vitsas, A. C. Boucouvalas, "Packet Delay Analysis of the IEEE 802.11 MAC Protocol," in IEE Electronic Letters, vol. 39, no. 18, 2003, pp. 13581359.

[13] T. Sakurai and Hai L. Vu, "MAC Access Delay of IEEE 802.11 DCF", in IEEE Transactions on Wireless Comm., Vol. 6, No. 5, May 2007, pp 1702-1710.

[14] Y. Li, C. Wang, K. Long, W. Zhao, "Modeling Channel Access Delay and Jitter of IEEE802.11 DCF," in Wireless Personal Communications, vol. 47, no. 3, November 2008, pp. $417-440$.
[15] Y. Chen and D. P. Agrawal, "Effect of Contention Window on the Performance of IEEE 802.11 WLANs", in Proceedings, 3rd Annual Mediterranean Ad Hoc Networking Workshop June 27-30, 2004.

[16] Y. Chen, Q-A Zeng and D. P. Agrawal. "Performance Analysis and Enhancement of IEEE 802.11 MAC Protocol," in Proceedings of IEEE International Conference on Telecommunications, 2003.

[17] D. Qiao, and K. Shin, "UMAV: A Simple Ehancement to the IEEE 802.11 DCF," in Proceedings of the $36^{\text {th }}$ Hawaii International Conference on System Sciences (HICSS-36), January 2003.

[18] "ns-2 Network Simulator," http://www.isi.edu/nsnam/ns/. 